\title{
Pressure Stability under a Pump Failure*
}

\author{
S. A. Heifets, ${ }^{\star}$ J. Seeman, ${ }^{\star}$ and W. Stoeff ${ }^{\diamond}$ \\ *Stanford Linear Accelerator Center, Stanford University, Stanford, CA 94309 USA \\ ^Lawrence Livermore Laboratory, Livermore, CA 94550 USA
}

Ions produced by a beam on the residual gas induce desorption from the beam pipe wall and may lead to a runaway pressure build up. The main mechanism of ion production is usually inelastic collisions of the beam particles. It may not be true for PEP-II where the combination of high energy and high beam current leads to MWs of the total power $P_{0}$ in synchrotron radiation. The photoeffect on the residual gas may produce more ions than produced in the inelastic collisions due to a much larger cross-section of the photoeffect $\sigma^{\gamma}$ at low photon energies $h \omega$ where the number of photons $d P(\omega) / h \omega$ is maximum.

The total cross-section $\sigma_{t}=(1+\Delta) \sigma_{e}$, where $\sigma_{e}$ is the cross-section of the inelastic collision and correction $\Delta$, which is the ratio of number of ions produced by photoeffect to the number of ions produced in inelastic collisions, can be estimated as

$$
\Delta=\frac{N^{\gamma}}{N^{e}}=0.84 \alpha \gamma \sqrt{\frac{b}{\rho}} \int \frac{d \omega}{\omega}\left(\frac{\omega}{\omega_{c}}\right)^{1 / 3} \frac{\sigma^{\gamma}(\omega)}{\sigma^{e}} .
$$

Here $\omega_{c}$ is the critical frequency of the synchrotron radiation, and $\alpha=1 / 137$.

The cross-section of the photoeffect on a K-shell electron of a hydrogen-like atom with the charge $Z$ is well known. To describe the low-energy photoeffect we scale it according to the Thomas-Fermi model, replacing parameters of a hydrogen-like atom by the parameters of an atom with the ionization potential $I_{0}$. That gives

$$
\sigma^{\gamma}=0.23 Z \frac{a_{0}^{2}}{Z^{2 / 3}}\left(Z^{4 / 3} \frac{I_{0}}{h \omega}\right)^{4} \frac{\exp \{-4[\nu \operatorname{arccot} \nu-1]\}}{1-\exp \{-2 \pi \nu\}},
$$

where $I=Z^{2} I_{0}, \nu=(h \omega / I-1)^{1 / 2}, I_{0}=13.6 \mathrm{eV}$, and $a_{0}=0.5 \times 10^{-8} \mathrm{~cm}$ are parameters of a hydrogen atom. Numerical calculations give

$$
\int \frac{d \omega}{\omega}\left(\frac{\omega}{\omega_{c}}\right)^{1 / 3} \frac{\sigma^{\gamma}(\omega)}{\sigma^{e}}=0.094 Z^{7 / 9}\left[\frac{I_{0}}{h \omega_{c}}\right]^{1 / 3}\left(\frac{a_{0}^{2}}{\sigma_{e}}\right) .
$$

For the parameters of the PEP-II HER and $Z=28$, $\Delta=1.35$, and the total cross-section is larger than the inelastic cross-section by the factor 2.35 .

\footnotetext{
*Work supported by Department of Energy contract DE-AC03-76SF00515 (SLAC).
}

The pressure $P(z)$ along the pipe in the straight sections is found to be

$$
P(z)=\frac{q_{s r} L}{4 W} \frac{1}{\psi^{2}}\left[-1+\frac{\cos (\Omega z-\psi)}{\cos \psi-(2 W / S) \psi \sin \psi}\right],
$$

where $q_{s r}$ is the ion induced outgasing rate per unit length in (torr $1 / \mathrm{m} / \mathrm{sec}$ ) induced by synchrotron radiation, $q_{i}=$ $\eta \sigma_{t}(I / e)$ is the outgasing induced by the ions produced in collisions with the residual gas, $I$ is the average beam current, $\psi=\Omega L / 2, \Omega=\sqrt{q_{i} / L W}, L$ is the pump separation, $S$ is the pumping speed in $(1 / \mathrm{sec})$, and $W$ is the pipe conductance in $(1 / \mathrm{sec})$. The desorption coefficient $\eta$, the number of outgased molecules per ion, depends on the ion mass, energy, material and treatment of the wall, and can change in the wide range from $\eta \simeq 0.01$ to $\eta \simeq 10$.

Equation 4 shows that $P(z)$ goes to infinity if $\psi \tan \psi=S / 2 W$, defining the threshold current $I_{t h}$ at which pressure instability takes place. For the parameters: $\sigma^{E}=2 \times 10^{-18} \mathrm{~cm}^{2}, W=24.8 \mathrm{l} / \mathrm{sec}, S=68 \mathrm{l} / \mathrm{sec}$, and $\eta I_{\text {th }}=10.47 \mathrm{~A}$. Figure 1 shows the pressure profile for $\eta=1$ and the current in the range from $1 \mathrm{~A}$ to $9 \mathrm{~A}$.

Consider now a situation when a pump at $z=0$ fails doubling the pumping distance. The pressure profile in this case for the range $-L<z<L$ is

$$
P(z)=\frac{q_{s r} L}{4 W} \frac{1}{\psi^{2}}\left[-1+\frac{\cos (\Omega z) \cos \psi}{\cos 2 \psi \cos \psi-\frac{W}{S} \psi \sin 3 \psi}\right],
$$

giving the maximum pressure at $z=0$.

The solution describes substantial increase of the pressure at $z=0$ (by a factor $\simeq 4$ ) compared to Eq. (4) and predicts the runaway situation at the current defined by

$$
\psi \frac{\sin 3 \psi}{\cos 2 \psi \cos \psi}=\frac{S}{W}
$$

The lowest root of this equation defines the threshold current

$$
\frac{L}{W} \eta I_{t h} \sigma_{c}=6.4 \psi^{2}
$$

where $L$ is in meters, $W$ is in $1 / \mathrm{sec}, I_{t h}$ in ampers, and $\sigma_{c}$ is in units $10^{-18} \mathrm{~cm}^{2}$. This function is shown in Fig. 2 for the normal case (low curve) and with pump failure (upper curve). The right-hand side goes to a maximum value of 3.9 at large $S / W$ giving $\eta I_{\text {th }}=4.96 \mathrm{~A}$ for the

\footnotetext{
- Presented at the 16th IEEE Particle Accelerator Conference (PAC 95) and International Conference on High-Energy Accelerators, Dallas, Texas, May 1-5, 1995

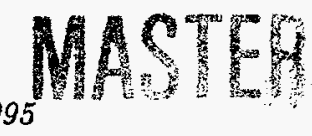




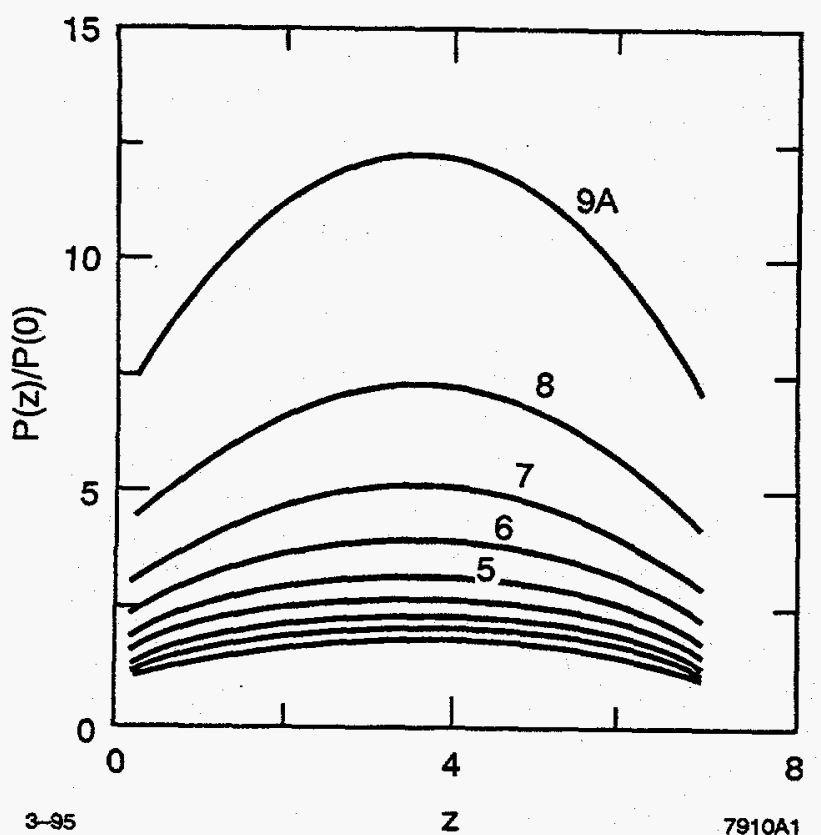

Figure 1. Pressure profile between pumps separated by 7 $\mathrm{m}$ for the beam current from $1 \mathrm{~A}$ to $9 \mathrm{~A}$. The ion induced threshold current is $I_{t h}=10.469 \mathrm{~A}$ for $W=24.8 \mathrm{l} / \mathrm{sec}$, and $S=68 \mathrm{l} / \mathrm{sec}$.

pipe $r=5 \mathrm{~cm}, L=7 \mathrm{~m}$, and $\sigma_{c}=210^{-18} \mathrm{~cm}^{2}$. The threshold current is reduced from $10.47 \mathrm{~A}$ to $4.75 \mathrm{~A}$ for the parameters used above.

The conductance calculated from local conductances (M. Sullivan, private communication) is $W=84 \mathrm{l} / \mathrm{s}$ and $S=400 \mathrm{l} / \mathrm{s}$ for the interaction region $\pm 2.45 \mathrm{~m}$ from IP. That gives quite high $\eta I_{t h}=27.4 \mathrm{~A}$.

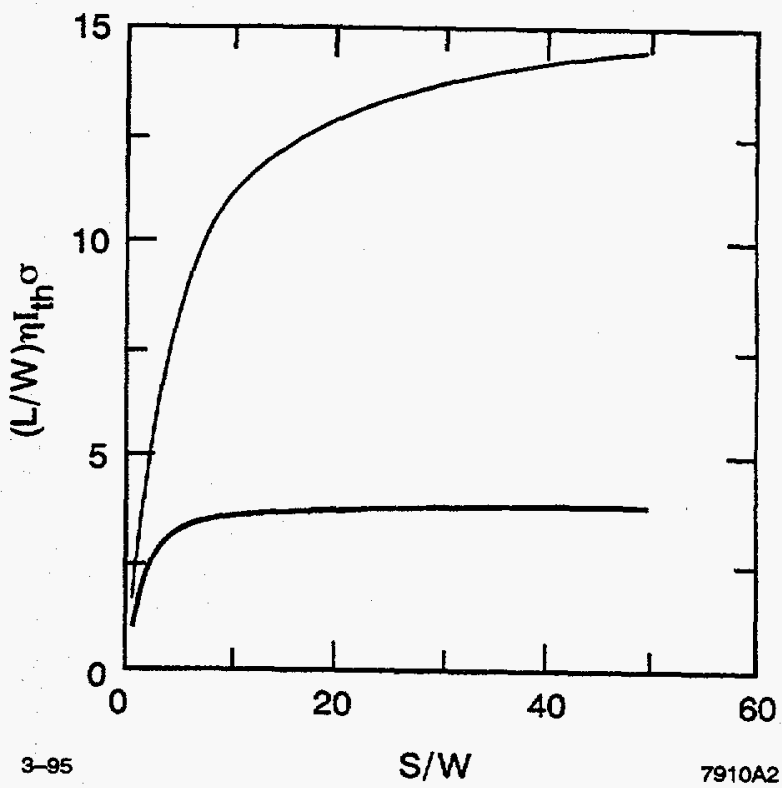

Figure 2. Parameter in the LHS of Eq. (7) versus $S / W$.

The threshold current is given by the pumping speed $s$ of the distributed ion pumps for the HER arcs: $\eta I_{t h} \sigma_{c}=$ $1.6 * s$ where $s$ is in $l / \mathrm{sec}, \sigma_{c}$ is in $10^{-18} \mathrm{~cm}^{2}$, and $I_{t h}$ in $A$ is very high for $s=120 \mathrm{l} / \mathrm{m} / \mathrm{sec}$.

The situation is less obvious for the wiggler vacuum chamber (under design).

The estimate shows that PEP-II should not have a problem with a pressure instability at nominal pumping speed provided that $\eta$ remains small, $\eta<1$.

\section{DISCLAIMER}

This report was prepared as an account of work sponsored by an agency of the United States Government. Neither the United States Government nor any agency thereof, nor any of their makes any warranty, express or implied, or assumes any legal liability or responsibility for the accuracy, completeness, or usefulness of any information, apparatus, product, or bility for thelosed, or represents that its use would not infringe privately owned rights. Refer-

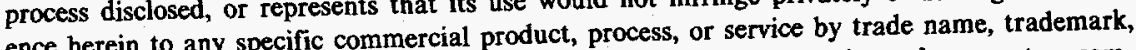
ence herein to any specis ot nerwise does not necessarily constitute or imply its endorsement, recommanufacturer, or otherwise does not necessarily constitute or imply its endorent mendation, or favoring by the United States Governmessily state or reflect those of the and opinions of authors expressed herein do not 


\section{DISCLAIMER}

Portions of this document may be illegible in electronic image products. Images are produced from the best available original document. 